\title{
Novel concepts of antiangiogenic therapies in metastatic renal cell cancer
}

\author{
Renate Pichler · Isabel Heidegger
}

Received: 31 March 2017 / Accepted: 11 July 2017 / Published online: 11 August 2017

(C) The Author(s) 2017. This article is an open access publication.

Summary The era of antiangiogenic drugs targeting the vascular endothelial growth factor (VEGF) signaling pathway has become a mainstay in the treatment of metastatic renal cell carcinoma (mRCC), showing primary responses in $65-70 \%$ of patients. Nevertheless, most of those patients progress to angiogenesis inhibitors over time due to different modes of resistance (adaptive and intrinsic). Both in vitro and in vivo analyses provided evidence that PD-L1 upregulation in hypoxia conditions is dependent on hypoxiainducible factor (HIF)-2alpha and is associated with an overexpression of VEGF. Thus, additional blockade of PD-L1 along with inhibition of angiogenesis pathways seems to represent a novel and innovative treatment concept in mRCC. In this short review, we provide an overview on ongoing phase III trials combining antiangiogenic therapies with checkpoint inhibitors in the first-line setting. Moreover, we critically analyze the impact of recently approved therapeutic antiangiogenic agents and checkpoint inhibitors after progression to first-generation tyrosine kinase inhibitors and their mode of action. In addition, response and resistance hypotheses and biomarkers to antiangiogenic therapy in clinical practice are critically discussed.

Keywords Renal cell carcinoma - Antiangiogenic therapy · Programmed-death ligand 1 - Hypoxia · Resistance mechanism

R. Pichler, MD PhD $(\bowtie) \cdot$ I. Heidegger, MD PhD

Department of Urology, Medical University Innsbruck, Anichstraße 35, 6020 Innsbruck, Austria

Renate.Pichler@i-med.ac.at

I. Heidegger, $\mathrm{MD} \mathrm{PhD}$

Isabel-Maria.Heidegger@i-med.ac.at
Renal cell carcinoma (RCC) is the ninth most common cancer worldwide, with approximately 63,990 estimated new cases in 2017 in the United States [1]. Approximately $20-30 \%$ of patients present in a metastatic stage at the time of diagnosis. Approximately one third of those patients with initial curative surgical approach will develop local recurrence or distant metastases over time [2, 3]. Clear cell renal cell carcinoma (ccRCC), a subtype of RCC, is a highly vascularized tumor and is therefore an attractive disease to study angiogenesis and to test novel angiogenesis inhibitors in early clinical development. The introduction of vascular endothelial growth factor (VEGF)-targeted tyrosine kinase inhibitors (TKIs) 10 years ago has revolutionized the systemic treatment of metastatic RCC (mRCC) after the cytokine decade using interleukin-2 (IL-2) and interferon-alpha (IFN-alpha) [4]. Nevertheless, complete response is confirmed in less than $1 \%$ as most patients with initial response progress during antiangiogenic therapy due to diverse resistance mechanisms $[2,4]$.

\section{Biology of resistance and response hypotheses to antiangiogenic agents}

In general, two different modes of resistance to antiangiogenic agents, the adaptive (evasive) and the intrinsic (pre-existing) non-responsiveness, have been described in preclinical models $[5,6]$. The adaptive resistance is built on the concept that angiogenic tumors can develop an adaptation to VEGF-targeted therapy by evading the therapeutic blockade of angiogenesis due to an upregulation of alternative angiogenic and invasive pathways, including MET and AXL (receptor tyrosine kinases) [4, 5]. For example, it is well known that a chronic sunitinib therapy in RCC cell lines can induce MET and AXL signaling, thus promoting the epithelial-mesenchymal transi- 


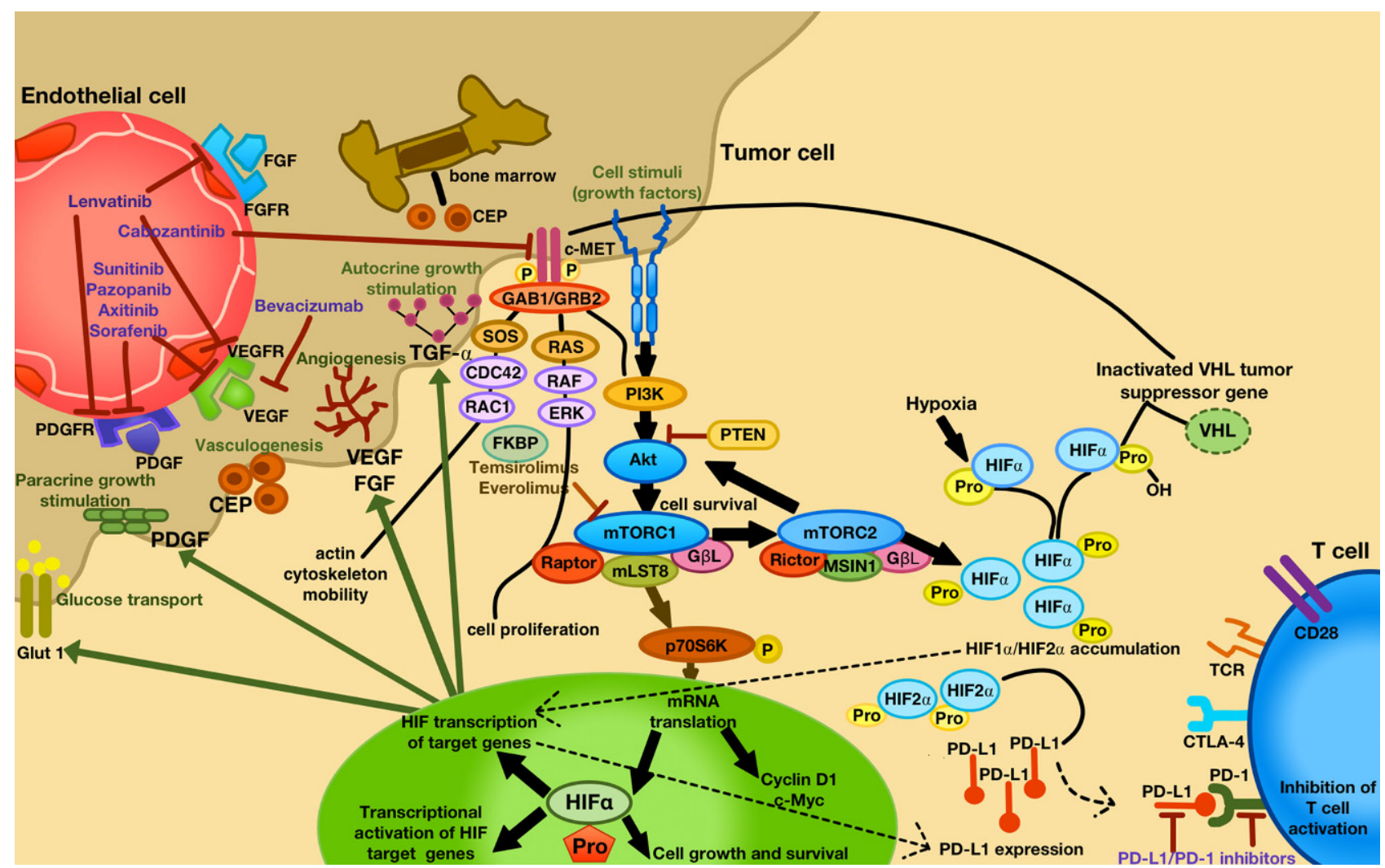

Fig. 1 Approved VEGF-targeted and mTOR-targeted antiangiogenic drugs and their specific targets with their mode of action. Increased tumor hypoxia during antiangiogenic therapy is the key player for developing TKI resistance, with an accumulation of HIF-alpha. Consequently, different alternative HIF and/or non HIF-derived proangiogenic (e. g. ephrin, angiopoietin, FGF, VEGF, PIGF) and c-MET (cell motility, proliferation, differentiation, migration and invasion) signaling pathways are activated, being responsible for further tumor progression. More-

tion (EMT), with increased cell invasion, migration and angiogenesis [7]. Moreover, Von-Hippel-Lindau (VHL) mutations in ccRCC patients with antiangiogenic therapies induce a hypoxia-inducible factor (HIF)alpha accumulation, also activating alternative HIF and/or non HIF-mediated proangiogenic signaling pathways in the tumor, such as fibroblast growth factor (FGF), placental growth factor (PIGF), ephrin and angiopoietin [5]. In addition, a recruitment of bone marrow-derived cells consisting of vascular progenitors and proangiogenic monocytic cells for vasculogenesis is induced, limiting the obligatory necessity of VEGF signaling [8]. Another consequence of upregulated alternative angiogenic pathways is an increase of pericyte coverage for protecting tumor blood vessels [5] and tumor cell invasiveness to escape oxygen deprivation [5]. Moreover, a compensatory increase of the phosphatidylinositide 3-kinase (PI3K) and protein kinase B (Akt/PKB) pathway due to mammalian target of rapamycin complex (mTORC) 1 inhibition (everolimus, temsirolimus) may lead to an upregulation of mTORC2 with further Akt und HIF activation; however, whether mTOR inhibitors that target both over, hypoxia leads to an activation of bone marrow-derived cells consisting of circulating endothelial progenitor cell (CEP), forming new blood vessels in the tumor (vasculogenesis). Under hypoxia, PD-L1 upregulation was dependent on HIF-2a in RCC, being associated with simultaneous VEGF overexpression. CEP circulating endothelial progenitor, $F G F(R)$ fibroblast growth factor (receptor), PDGF (R) platelet-derived growth factor (receptor), PIGF placental growth factor, VEGF (R) vascular endothelial growth factor (receptor)

mTOR complexes increase antitumor effects has yet to be tested in RCC ([9]; Fig. 1).

The intrinsic resistance to antiangiogenic therapies is explained by the fact that certain tumors have a preexisting resistance, meaning that tumors have already activated evasive resistance mechanisms, before starting antiangiogenic therapy in response to the selective mechanisms within the microenvironment during pre-malignant transformation [5].

A major challenge in clinical practice, is to elucidate potential predictive biomarkers identifying those patients who mostly benefit from a certain antiangiogenic agents [10]. It has been previously reported that response or resistance to antiangiogenic agents may be evaluated by endothelial cell effects, such as therapy-induced hypertension [11], treatment-induced functional radiographic changes in tumor blood flow by dynamic contrast-enhanced magnetic resonance imaging (DCE-MRI) $[10,12]$ or by measuring levels of circulating endothelial cells (CEC), circulating endothelial progenitor cells (CEP) and tumor endothelial markers [10]. Another approach to overcome angiogenic escape may be a rechallenge of antiangiogenic 


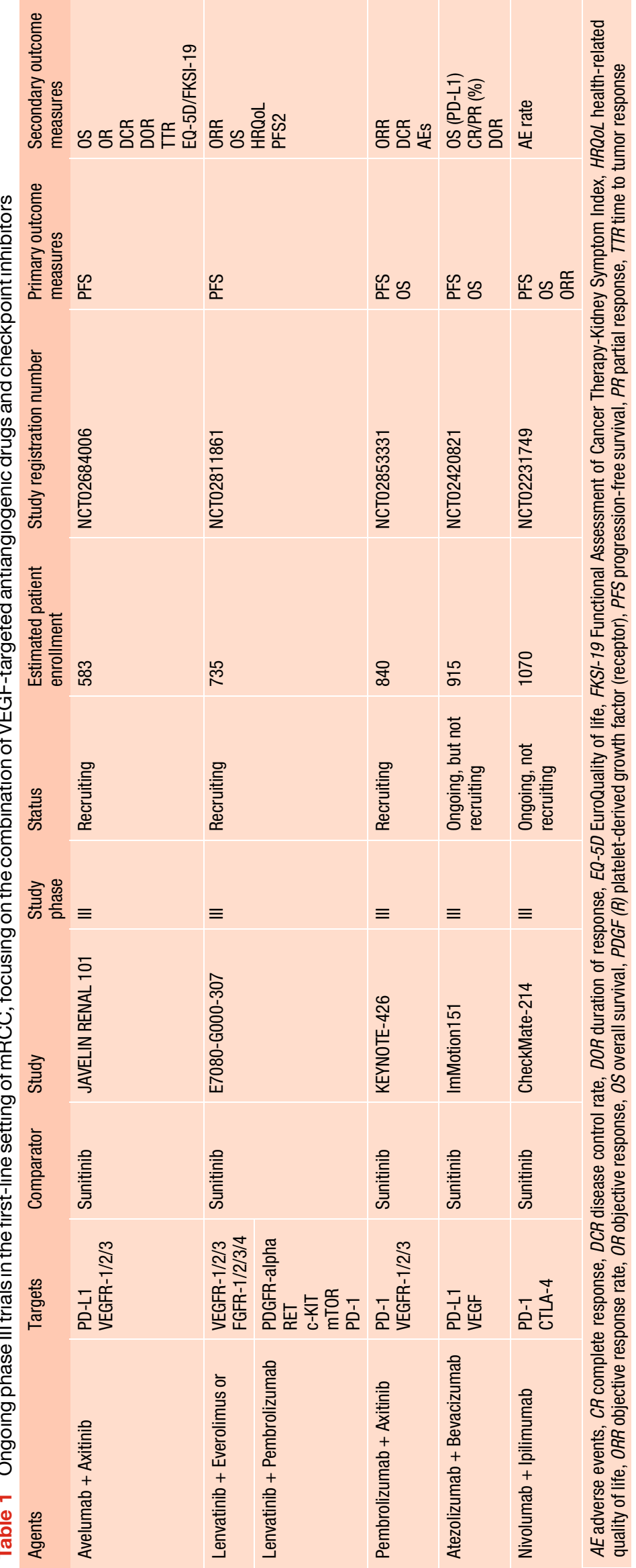


Fig. 2 Schematic overview of the current European Association of Urology 2017 guidelines and evidencebased recommendations for systemic treatment in mRCC (level of evidence). OS overall survival, PFS progression-free survival, VEGF vascular endothelial growth factor, mTOR mammalian target of rapamycin. (Adapted from [22])

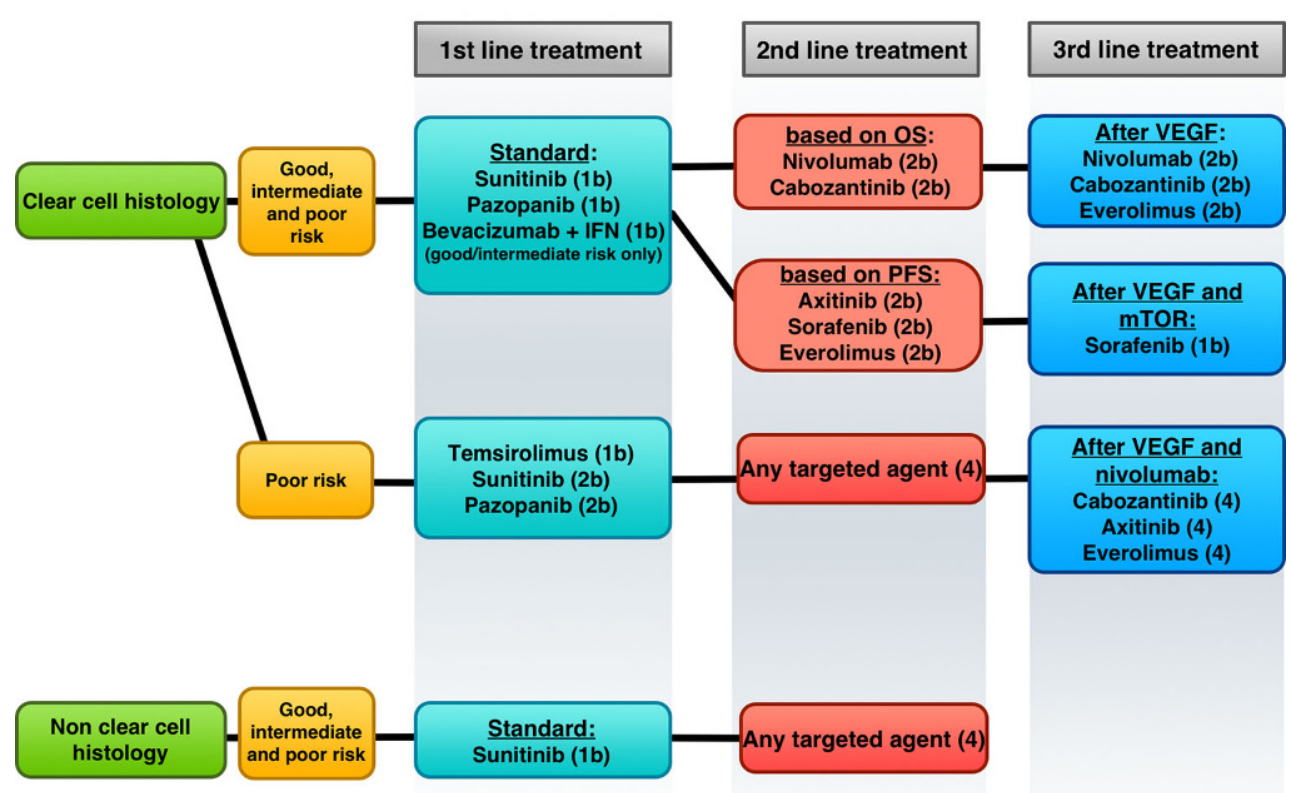

drugs due to inadequate target inhibition based on increased receptor signaling and/or reduced drug levels [9]. Nevertheless, only few trials tested a comprehensive biomarker panel of possible resistance/ response mechanisms during antiangiogenic therapy in the clinical setting $[10,13]$. Thus, further validation of these preliminary results is obligatory before drawing any final conclusions.

Tumor hypoxia and PD-L1 expression: a novel therapeutic approach in the first-line setting in mRCC?

The VEGF-targeted antiangiogenic agents induce tumor hypoxia, leading to an upregulation of programmed death-ligand 1 (PD-L1) in different cancer entities including hepatocellular carcinoma [14], lung cancer [15] and RCC [16]. Generally, hypoxic zones in the tumor can attract different immunosuppressive myeloid cells, such as myeloid-derived suppressor cells (MDSC). Under hypoxic conditions, HIF-1alpha leads to an upregulation of PD-L1 expression on MDSCs in the tumor microenvironment, thereby increasing interleukin (IL)-6 and IL-10 secretion from MDSCs, causing a MDSC-induced immunosuppression, $\mathrm{T}$ cell inactivation, and promoting tumor progression [17, 18]. In RCC, hypoxia, and in addition, a loss of the VHL protein (pVHL) results in the constitutive stabilization of HIF1alpha and HIF2alpha, inducing various HIF-transcriptional targets [19]. The PD-L1 as a HIF2alpha target was upregulated in VHL protein deficient ccRCC in vitro [20]. In RCC patients, PD-L1 expression positively correlated with VHL mutation, HIF-2alpha expression, adverse pathological features such as higher nuclear grade, necrosis and sarcomatoid transformation, c-MET and VEGF ex- pression [16, 21], thus resulting in a shorter progression-free and cancer-specific survival [21]. Based on these data, simultaneous blockade of PD-L1 with the inhibition of the VHL/HIF/VEGF pathway may represent a novel and innovative treatment concept [17]. Thus, various randomized phase III trials in the first-line setting of mRCC are currently ongoing, testing this combined therapeutic approach consisting of checkpoint inhibitors (avelumab, pembrolizumab, atezolizumab, nivolumab, ipilimumab) combined with VEGF-targeted antiangiogenic agents (axitinib, lenvatinib, bevacizumab) in comparison to standard first-line drugs alone (sunitinib) (Table 1). Results of these trials are expected soon.

Novel approved TKIs and checkpoint inhibitors in $\mathrm{mRCC}$ after progression to first-generation VEGF-targeted antiangiogenic agents

In 2016, the Food and Drug Administration (FDA) and the European Medicines Agency (EMA) have approved to two novel TKIs, cabozantinib and lenvatinib (in combination with the mTOR inhibitor everolimus) and one PD-1 inhibitor, nivolumab, after progression to first-generation VEGF-targeted TKIs in mRCC, changing and radically improving the sequence therapy in the second-line, third-line and in the later line setting. A schematic overview of the current European Association of Urology (EAU) 2017 guidelines with evidence-based recommendations for systemic treatment in mRCC is shown in Fig. 2.

Cabozantinib This is an oral multi-TKI blocking VEGFR-1, 2, 3, RET, KIT, TRKB, FLT-3, AXL, TIE-2, with the additional potential to inhibit c-MET. The c-MET expression was noticed to be an independent 
prognostic marker and a potential therapeutic target especially in ccRCC, associated with worse diseasespecific survival [23] due to aggressive tumor behavior [24] and increased PD-L1 expression [21]. The openlabel, randomized phase III METEOR trial included 658 mRCC patients with previous treatment with one or more VEGF-targeted TKIs, receiving cabozantinib $60 \mathrm{mg}$ or everolimus $10 \mathrm{mg}$ once per day consecutively. Interestingly, the median progression-free survival (PFS) (cab vs. eve: 7.4 vs. 3.9 months; hazard ratio $\mathrm{HR}=0.51 ; p<0.0001)$ as well as the overall survival (OS) (cab vs. eve: 21.4 vs.16.5 months; HR = $0.66 ; p=0.00026$ ) was significantly increased in the cabozantinib group compared to everolimus, thus becoming one of the new efficient second-line TKIs in the recent European Society of Medical Oncology (ESMO) and EAU guidelines. Nevertheless, a dose reduction during treatment occurred in $60 \%$ of all patients undergoing cabozantinib therapy, with serious adverse events grades $3 / 4$ in $39 \%$ [25].

Lenvatinib This is a multi-target TKI of VEGFR-1, -2, -3 also inhibiting FGFR-1, $-2,-3$ and -4, PDGFRalpha, KIT and RET. Antiangiogenesis activity and antitumor cell growth of lenvatinib was previously confirmed by inhibiting VEGF and FGF-driven proliferation and tube formation of human umbilical vein endothelial cells in vitro. In addition, in vivo angiogenesis induced by overexpressed VEGF or FGF was significantly suppressed with oral lenvatinib treatment [26]. The enhanced antitumor activity by combining lenvatinib plus everolimus may be explained by the simultaneous targeting of tumor cell growth and angiogenesis in human RCC xenograft models [27]. This combination confirmed an additive activity in VEGF-activated, and synergistic activity against FGF-activated endothelial cells, with suppression of mTOR-S6K-S6 signaling [27]. In the clinical setting, the phase 1b study confirmed lenvatinib $18 \mathrm{mg}$ and everolimus $5 \mathrm{mg}$ once a day as the maximum tolerated dose in patients with mRCC, with manageable toxicity and the best therapeutic response (stable disease in $45.5 \%$ and partial remission in $36.4 \%$ ) [28]. The following phase II trial with 153 patients who progressed after firstline VEGF-targeted therapy received either lenvatinib $18 \mathrm{mg}$ combined with everolimus $5 \mathrm{mg}$, single-agent lenvatinib $24 \mathrm{mg}$, or single-agent everolimus $10 \mathrm{mg}$. Compared to lenvatinib and everolimus monotherapy, the combination of lenvatinib and everolimus showed the best median PFS (14.6 months) and median OS (25.5 months), with diarrhea as the most common grade $3 / 4$ adverse event in $20 \%$ [29].

Based on the limited size of approximately 150 patients in this phase II study, the combination of lenvatinib and everolimus was not, at this stage, recommended either by current ESMO 2016 [30] or by EAU 2017 guidelines [22] on RCC as a novel second-line therapeutic regimen.
Nivolumab This is the first approved PD-1 checkpoint inhibitor in the second-line treatment of mRCC. The randomized phase II trial evaluated three doses of nivolumab $(0.3,2$ and $10 \mathrm{mg} / \mathrm{kg}$ intravenously once every 3 weeks) to identify a potential dose-response relationship and assess the activity and safety of nivolumab in patients with mRCC. Interestingly, no dose-dependent relationship was confirmed by PFS (2.7 vs. 4.0 vs. 4.2 months, respectively) and ORR ( $20 \%$ vs. $22 \%$ vs. $20 \%$, respectively) with manageable safety profiles across the three doses (grade 3-4 adverse events AE: $5 \%$ vs. $17 \%$ vs. $13 \%$, respectively) [31]. The following phase III Checkmate 025 trial compared nivolumab ( $3 \mathrm{mg} / \mathrm{kg}$ intravenously every 2 weeks) with everolimus ( $10 \mathrm{mg}$ orally once a day) in patients who received previous treatment with one or two regimens of antiangiogenic therapy. Nivolumab confirmed significantly better median OS (25.0 vs. 19.6 months) and ORR ( $25 \%$ vs. $5 \%$, OR $=5.98$ ) in comparison to everolimus [32]. In a further subgroup OS analyses of the Checkmate 025 study population, nivolumab confirmed an OS improvement versus everolimus across all subgroups including age, number of sites of metastases, type of metastases, number and duration of prior therapies, type of prior therapy, and Memorial Sloan Kettering Cancer Center (MSKCC) risk groups, with a high benefit in patients belonging to the poor MSKCC group [33]. Moreover, the rate of grade 3 or 4 AEs was less in patients treated with nivolumab (19\%) compared to everolimus (37\%) [32], thus resulting in a significant improvement of health-related quality of life in patients treated with nivolumab versus everolimus (55\% vs. $37 \%, p<0.0001$ ) [34].

\section{Conclusion}

Angiogenic tumors can develop an adaptation to VEGF-targeted therapy by evading the therapeutic blockade of angiogenesis due to an upregulation of alternative angiogenic and invasive pathways. The VEGF-targeted antiangiogenic-induced tumor hypoxia leads to an upregulation of HIF1 and 2alpha, thus activating survival pathways in the tumor cells with an increased activation of proangiogenic signaling pathways. In addition, PD-L1 expression is upregulated by HIF-2alpha in RCC. Thus, the combined therapeutic approach simultaneously inhibiting the VHL/HIF/VEGF pathway and the PD-L1 expression seems to be an attractive and efficient method for increasing antitumor activity in mRCC. Several phase III clinical trials are currently investigating the combination of TKIs plus immunotherapy compared to TKI alone in the first-line setting, the results of which are expected soon.

Acknowledgements Open access funding provided by University of Innsbruck and Medical University of Innsbruck.

Conflict of interest R. Pichler and I. Heidegger declare that they have no competing interests. 
Open Access This article is distributed under the terms of the Creative Commons Attribution 4.0 International License (http://creativecommons.org/licenses/by/4.0/), which permits unrestricted use, distribution, and reproduction in any medium, provided you give appropriate credit to the original author(s) and the source, provide a link to the Creative Commons license, and indicate if changes were made.

\section{References}

1. Siegel RL, Miller KD, Jemal A. Cancer statistics. CA Cancer J Clin. 2017;67(1):7-30. doi:10.3322/caac.21387.

2. Gupta K, Miller JD, Li JZ, Russell MW, et al. Epidemiologic and socioeconomic burden of metastatic renal cell carcinoma (mRCC): A literature review. Cancer Treat Rev. 2008;34(3):193-205. doi:10.1016/j.ctrv.2007.12.001.

3. Cindolo L, Patard JJ, Chiodini P, et al. Comparison of predictive accuracy of four prognostic models for nonmetastatic renal cell carcinoma after nephrectomy: A multi-center European study. Cancer. 2005;104(7):1362-71.

4. Tannir NM, Schwab G, Grünwald V. Cabozantinib: an active novel multikinase inhibitor in renal cell carcinoma. Curr OncolRep. 2017;19:14.

5. Bergers G, Hanahan D. Modes of resistance to anti-angiogenic therapy. Nat Rev Cancer. 2008;8(8):592-603. doi:10. 1038/nrc2442.

6. Welti J, Loges S, Dimmeler S, Carmeliet P. Recent molecular discoveries in angiogenesis and antiangiogenic therapies in cancer. J Clin Invest. 2013;123(8):3190-200. doi:10.1172/ JCI70212.

7. Zhou L, Liu XD, Sun M, et al. Targeting MET and AXL overcomes resistance to sunitinib therapy in renal cell carcinoma. Oncogene. 2015;35(21):2687-97.

8. Shaked Y, Henke E, Roodhart JM, Mancuso P, et al. Rapid chemotherapy-induced acute endothelial progenitor cell mobilization: Implications for antiangiogenic drugs as chemosensitizing agents. Cancer Cell. 2008;14(3):263-73. doi:10.1016/j.ccr.2008.08.001.

9. Rini BI. New strategies in kidney cancer: therapeutic advances through understanding the molecular basis of response and resistance. Cancer Res. 2010;16(5):1348-54. doi:10.1158/1078-0432.CCR-09-2273.

10. Pircher A, Jöhrer K, Kocher F, et al. Biomarkers of evasive resistance predict disease progression in cancer patients treated with antiangiogenic therapies. Oncotarget. 2016;7(15):20109-23. doi:10.18632/oncotarget.7915.

11. Biomarkers RBI. hypertension following anti-angiogenesis therapy. Clin Adv Hematol Oncol. 2010;8(6):415-6, Jun.

12. de Bazelaire C, Alsop DC, George D, et al. Magnetic resonance imaging-measured blood flow change after antiangiogenic therapy with PTK787/ZK 222584 correlates with clinical outcome in metastatic renal cell carcinoma. Clin Cancer Res. 2008;14:5548-54.

13. Nikolinakos PG, Altorki N, Yankelevitz D, et al. Plasma cytokine and angiogenic factor profiling identifies markers associated with tumor shrinkage in early-stage non-small cell lung cancer patients treated with pazopanib. Cancer Res. 2010;70(6):2171-9. doi:10.1158/0008-5472.CAN-092533.

14. Semaan A, Dietrich D, Bergheim D, et al. CXCL12 expression and PD-L1 expression serve as prognostic biomarkers in HCC and are induced by hypoxia. Virchows Arch. 2017;470(2):185-96. doi:10.1007/s00428-016-2051-5.

15. Yang CY, Lin MW, Chang YL, Wu CT, Yang PC. Programmed cell death-ligand 1 expression is associated with a favourable immune microenvironment and better overall survival in stage I pulmonary squamous cell carcinoma. Eur JCancer. 2016;57:91-103. doi:10.1016/j.ejca.2015.12.033.

16. Messai Y, Gad S, Noman MZ, et al. Renal cell carcinoma programmed death-ligand 1, a new direct target of hypoxiainduciblefactor-2 Alpha, is regulated by von Hippel-Lindau gene mutation status. Eur Urol. 2016;70(4):623-32. doi:10. 1016/j.eururo.2015.11.029.

17. Noman MZ, Desantis G, Janji B, et al. PD-L1 is a novel direct target of HIF- $1 \alpha$, and its blockade under hypoxia enhanced MDSC-mediated T cell activation. J Exp Med. 2014;211(5):781-90. doi:10.1084/jem.20131916.

18. Noman MZ, Chouaib S. Targeting hypoxia at the forefront of anticancer immune responses. Oncoimmunology. 2015;3(12):e954463, eCollection 2014.

19. Krieg M, Haas R, Brauch H, Acker T, Flamme I, Plate KH. Up-regulation ofhypoxia-induciblefactors HIF-lalphaand HIF-2alpha under normoxic conditions in renal carcinoma cells by von Hippel-Lindau tumor suppressor gene loss of function. Oncogene. 2000;19(48):5435-43.

20. Ruf M, Moch H, Schraml P. PD-L1 expression is regulated by hypoxia inducible factor in clear cell renal cell carcinoma. IntJCancer. 2016;139(2):396-403. doi:10.1002/ijc.30077.

21. Shin SJ, Jeon YK, Kim PJ, et al. Clinicopathologic analysis of PD-L1 and PD-L2 expression in renal cell carcinoma: Association with oncogenic proteins status. Ann Surg Oncol. 2016;23(2):694-702.

22. Ljungberg B, Albiges L, Bensalah K, Bex A, Giles RH, Hora M, KuczykMA, Lam T, Marconi L, Merseburger AS, Powles T, Staehler M, VolpeA, Dabestani S, Fernandez-Pello Montes S, Hofmann F, Tahbaz R. EAU Guidelines Panel. Guidelines on Renal Cell Carcinoma. https://uroweb.org/guideline/ renal-cell-carcinoma/. Accessed: 29.03.2017

23. Gibney GT, Aziz SA, Camp RL, et al. c-Met is a prognostic marker and potential therapeutic target in clear cell renal cell carcinoma. Ann Oncol. 2013;24(2):343-9. doi:10.1093/ annonc/mds463.

24. Macher-Goeppinger S, Keith M, Endris V, et al. MET expression and copy number status in clear-cell renal cell carcinoma: prognostic value and potential predictive marker. Oncotarget. 2017;8(1):1046-57. doi:10.18632/ oncotarget.13540.

25. ChoueiriTK, Escudier B, PowlesT, etal. Cabozantinibversus everolimus in advanced renal cell carcinoma (METEOR): final results from a randomised, open-label, phase 3 trial. Lancet Oncol. 2016;17(7):917-27. doi:10.1016/S14702045(16)30107-3.

26. Yamamoto Y, Matsui J, Matsushima T, et al. Lenvatinib, an angiogenesis inhibitor targeting VEGFR/FGFR, shows broad antitumor activity in human tumor xenograft models associated with microvessel density and pericyte coverage. Vasc Cell. 2014;6:18, eCollection 2014 doi:10.1186/2045824X-6- 18.

27. Matsuki M, Adachi Y, Ozawa Y, et al. Targeting of tumor growth and Angiogenesis underlies the enhanced Antitumor activity of Lenvatinib in combination with Everolimus. Cancer Sci. 2017; doi:10.1111/cas.13169.

28. Molina AM, Hutson TE, LarkinJ, etal. A phase 1bclinical trial of the multi-targeted tyrosine kinase inhibitor lenvatinib (E7080) in combination with everolimus for treatment of metastatic renal cell carcinoma (RCC). Cancer Chemother Pharmacol. 2014;73(1):181-9. doi:10.1007/s00280-0132339-y.

29. Motzer RJ, Hutson TE, Glen H, et al. Lenvatinib, everolimus, and the combination in patients with metastatic renal cell carcinoma: Arandomised, phase 2, open-label, multicentre trial. Lancet Oncol. 2015;16(15):1473-82. doi:10.1016/ S1470-2045(15)00290-9. 


\section{short review}

30. Escudier B, Porta C, Schmidinger M, et al. Renal cell carcinoma: ESMO Clinical Practice Guidelines for diagnosis, treatment and follow-up. Ann Oncol. 2016;27(suppl 5):v58-v68.

31. Motzer RJ, Rini BI, McDermott DF, et al. Nivolumab for metastatic renal cell carcinoma: Results of a randomized phase II trial. J Clin Oncol. 2015;33(13):1430-7. doi:10. 1200/JCO.2014.59.0703.

32. Motzer RJ, Escudier B, McDermottDF, et al. Nivolumab versus Everolimus in advanced renal-cell carcinoma. N Engl J Med. 2015;373(19):1803-13. doi:10.1056/NEJMoa1510665.

33. Escudier B, Sharma P, McDermott DF, et al. Checkmate 025 randomized phase 3 study: outcomes by key baseline factors and prior therapy for Nivolumab versus Everolimus in advanced renal cell carcinoma. Eur Urol. 2017;S0302-2838(17):30099-30094. doi:10.1016/j.eururo. 2017.02.010.
34. Cella D, GrünwaldV, Nathan P, et al. Quality oflifein patients with advanced renal cell carcinoma given nivolumab versus everolimusin CheckMate 025: A randomised, open-label, phase 3 trial. Lancet Oncol. 2016;17(7):994-1003. doi:10. 1016/S1470-2045(16)30125-5.

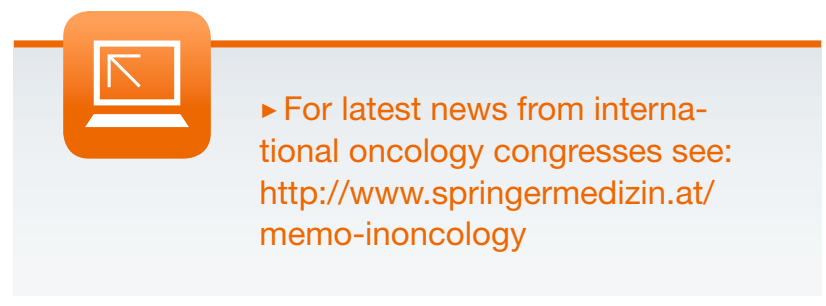

